\title{
ORIENTATIONAL BEHAVIOUR OF THE GUEST-HOST SYSTEMS IN THE SMECTIC A AND NEMATIC PHASES
}

\author{
D. Bauman, H. Moryson and E. Wolarz \\ Institute of Physics, Technical University, Piotrowo 3, 60-965 Poznani, Poland
}

(Received December 3, 1991)

\begin{abstract}
The measurements of the polarized absorption and fluorescence components spectra for the guest-host mixtures have been used to study the long orientational order in the uniaxial phases of the liquid crystal. The temperature dependence of the order parameters, $\left\langle P_{2}\right\rangle$ and $\left\langle P_{4}\right\rangle$, has been investigated and the molecular distribution function has been determined. On the basis of the $\left\langle P_{2}\right\rangle$ and $\left\langle P_{4}\right\rangle$, values some conclusions about the molecular orientation in the smectic $A$ and nematic phases of the liquid crystalline samples have been drawn. It has been also found that the fluorescence spectroscopy is a sensitive method for determination of the phase transition temperatures of the liquid crystal doped with the fluorescent dye.
\end{abstract}

PACS numbers: $61.30 . \mathrm{Gd}, 64.70 . \mathrm{Ew}$

\section{Introduction}

The studies of the molecular orientation of the pleochroic dye-liquid crystal mixtures (guest-host systems) are of interest of view of the technological applications of guest-host liquid crystal display devices, and are also of primary importance in understanding various aspects of the physics of liquid crystals as they give information about intermolecular forces in anisotropic media.

In a nematic phase of liquid crystal molecules are, on average, aligned with their long axes parallel to each other, what leads macroscopically to a preferred direction, described by the director $n$, and the centres of mass of the molecules are placed randomly. Therefore in this phase only a long-range orientational order occurs, which is usually determined by the order parameters $\left\langle P_{2}\right\rangle$ and $\left\langle P_{4}\right\rangle[1]$. A smectic phase is more highly ordered than the nematic one: it is characterized both by the orientational order of the long molecular axes and by a reduced positional order. The existence of a one-dimensional density wave is common to various smectic mesophases: the molecular centres of mass sit on planes perpendicular to 
the preferred axis, leading to a layer structure. Therefore a smectic A phase is characterized not only by the $\left\langle P_{L}\right\rangle$ parameters, but in addition by at least one density wave order parameter (translational order parameter) $\rho_{1}$ [2].

The translational order parameter for smectic $A$ can be determined only by measuring the integrated intensity of smectic $\mathrm{X}$-ray diffraction ring [3-5]. However, the orientational order parameters in the smectic $A$ phase could be evaluated by any of the methods used in the nematic phase, for example from the anisotropy of the refraction index, or by wide variety of spectroscopic methods, because both smectic $A$ and nematic phases are optically uniaxial, what has been considered by $\mathrm{X}$-ray diffraction pattern [4].

Experimental determination of the orientational order parameters for guest-host mixtures is usually carried out using the polarized absorption and/or fluorescence spectroscopies [6-15]. Previously, we had used the measurements of polarized absorption and fluorescence spectra of a pleochroic dye dissolved in some low molecular liquid crystals [14] and doped to side chain liquid crystal polymers $[13,15]$ in order to obtain information about the molecular orientation in the nematic phase. In this paper we have studied the long orientational order both in the nematic and smectic A phases for two mixtures of the $4^{\prime}$-n-octyl-4- $n$-cyanobiphenyl (8CB) doped with the fluorescent pleochroic dyes, derivatives of the bicarboxilic acid. Liquid crystal $8 \mathrm{CB}$, chosen for our studies, is of considerable importance, because it - similarly as other members of the alkyl-cyanobiphenyl homologous series, prepared by Gray et al. [16] - forms colourless, photochemically stable mesophases with strong positive dielectric anisotropy. Moreover 8CB is in the smectic A phase at the room temperature. To our knowledge the studies of the orientational order in the smectic phase has not been carried out up to now for guest-host mixtures.

\section{Experimental}

$\mathrm{M}$ a $\mathrm{t}$ e $\mathrm{r}$ i a $\mathrm{l}$ s: $8 \mathrm{CB}$ was supplied by BDH Ltd. and used without further purification: observed transition temperatures were in satisfactory agreement with those reported in literature [17-19] (see Table). As guest species the two bicarboxilic acid derivatives with the following chemical structure were used:

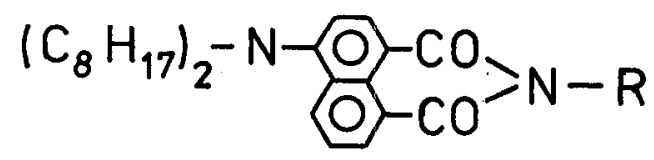

\begin{tabular}{c|c}
\hline \hline Dye code & $\mathrm{R}$ \\
\hline I & $-\mathrm{C}_{6} \mathrm{H}_{4}-\mathrm{O}-\mathrm{C}_{6} \mathrm{H}_{4}-\mathrm{CH}_{3}$ \\
II & $-\mathrm{C}_{6} \mathrm{H}_{4}-\mathrm{CSN}-\mathrm{C}_{6} \mathrm{H}_{3}-\mathrm{CH}_{3}$
\end{tabular}

They were synthesized and chromatographically purified in the Institute of Dyes, Lódź Technical University, Poland. The guests were dissolved in liquid crystal matrix at the concentration of $1.5 \times 10^{-3} \mathrm{M}$. 
A p p a r a t u s: The temperatures of the phase transitions for pure $8 \mathrm{CB}$ and doped with the dyes were determined by means of a polarizing microscope connected with a heating stage with the accuracy of $\pm 0.1^{\circ}$.

The absorption spectra were measured with a SPECORD M-40 spectrophotometer (Carl Zeiss Jena) equipped with polarizers. The polarized components of the fluorescence spectra were obtained in $\Pi$ geometry (Fig. 1) using a home-made photo-counting fluorimeter. The $436 \mathrm{~nm}$ line of the high pressured mercury lamp

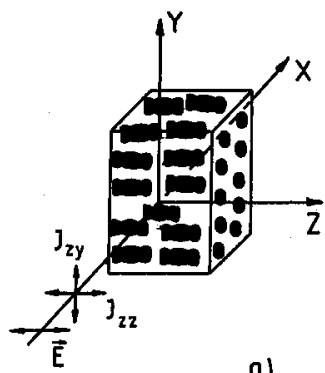

a)

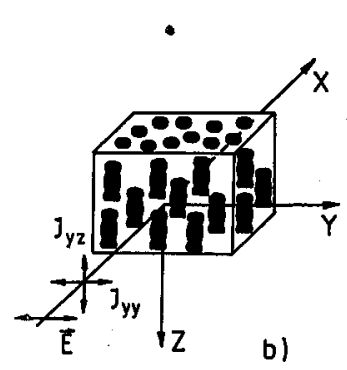

Fig. 1. Experimental geometries for measuring the emission of anisotropies $R_{1}$ (a) and $R_{2}$ (b). Arrows indicate the vibration direction of the electric vectors of the exciting and emitted light.

was used as an excitation source. Corrections for the photomultiplier spectral sensivity and for the different response of the device set with various directions of polarization were made. The measurements were carried out in the oriented "sandwich" cells of $20 \mu \mathrm{m}$ thickness. The temperature of the cells was regulated and controlled with the accuracy of $\pm 0.1^{\circ}$. The planar orientation of the liquid crystal and dye molecules had been achieved by treatment of the glass surfaces of the cells with polyimide and, additionally, by rubbing process. This procedure gives a good homogeneous orientation of the molecules in a thin layer, what has been controlled with the aid of crossed polarizers.

\section{Theory}

In the liquid crystalline state the molecules have a tendency to align their long axis along the direction of the director $n$. However, at finite temperature the thermal motion of molecules prevents perfect alignment with $n$ : the orientation of the molecules is in fact distributed in angle, but with the director as the most probable, or the most populated, direction. The orientation of any rod-like molecule in the director frame can be described using three Euler angles: $\varphi, \vartheta$, and $\psi$ shown in Fig. 2. Discussing the orientational order of a collection of molecules it is convenient to introduce an orientational distribution function $f(\varphi, \vartheta, \psi)$. Only uniaxial symmetry around the director is characterized for many liquid crystals, thus no order in the angle $\varphi$ (rotation in the azimuthal direction) is permitted. Moreover, if one assumes that the liquid crystal molecules are cylindrically symmetric, the rotation around the molecular symmetry axis (angle $\psi$ ) should not modify 


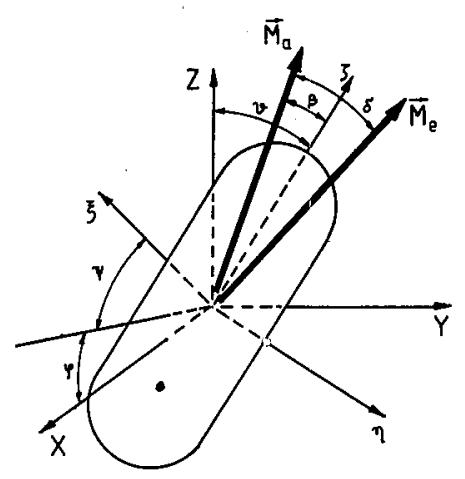

Fig. 2. Director frame $(X, Y, Z)$ and the principal axis frame $(\xi, \eta, \zeta)$ of molecule. $M_{\mathrm{a}}$ and $M_{\mathrm{e}}$ are directions of the absorption and emission transition moments, respectively.

the distribution function. Therefore the molecular distribution function $f(\vartheta)$ is dependent only on the angle $\vartheta$ between the director $n$ and the long molecular axis. Knowledge of $f(\vartheta)$ is of primary importance in liquid crystal research, because it completely describes the long-range orientational order which gives these substances their unique physical properties.

The orientational distribution function of the uniaxial liquid crystalline phase can be described as follows [7, 16-17]:

$$
f(\beta)=\sum_{L=0}^{\infty} \frac{2 L+1}{2}\left\langle P_{L}(\cos \beta)\right\rangle P_{L}(\cos \beta),
$$

where $P_{L}(\cos \vartheta)$ is the $L$-th Legendre polynomial. In the uniaxial mesophases, the experiments on most materials demonstrate that the heads or tails of the molecules are not distinguished in the molecular structure, thus only even terms can appear in Eq. (1). The first few terms of the expansion including $\left\langle\cos ^{2} \vartheta\right\rangle$ and $\left\langle\cos ^{4} \vartheta\right\rangle$ are

$$
\begin{aligned}
& \left\langle P_{0}\right\rangle=1, \\
& \left\langle P_{2}\right\rangle=\frac{1}{2}\left(3\left\langle\cos ^{2} \beta\right\rangle-1\right), \\
& \left\langle P_{4}\right\rangle=\frac{1}{8}\left(35\left\langle\cos ^{4} \beta\right\rangle-30\left\langle\cos ^{2} \beta\right\rangle+3\right) .
\end{aligned}
$$

The average of the second Legendre polynomial, $\left\langle P_{2}\right\rangle$, is equivalent to the liquid crystal order parameter, introduced by Zvetkoff [18] and usually reported as $S .\left\langle P_{2}\right\rangle=1$ for the completely ordered phase and $\left\langle P_{2}\right\rangle=0$ for the disordered isotropic phase. $\left\langle P_{4}\right\rangle$, however, contains further information about the orientational order and should in fact be more sensitive to molecular fluctuations because of the higher powers of the deviation angle. $\left\langle P_{4}\right\rangle$ could, therefore, be termed the "hyperorder" parameter. The direct physical meaning of the higher order terms of the series is hitherto not clear.

Most of the experimental methods which are used to determine the long orientational order in a liquid crystal (optical birefringence and diamagnetic anisotropy measurements, infrared spectroscopy, dichroism in visible and UV region) 
allow to estimate only the parameter $\left\langle P_{2}\right\rangle$. Both $\left\langle P_{2}\right\rangle$ and $\left\langle P_{4}\right\rangle$ can be simultaneously obtained on the basis of X-ray diffraction [19-23], magnetic resonance [25], Raman spectroscopy [25-32] as well as fluorescence depolarization of the dye dissolved in liquid crystalline matrix [7, 10-15].

The only difference between the Raman scattering and the emission of the fluorescence occurs on the scale of time: the Raman effect is almost instantaneous $\left(\approx 10^{-12} \mathrm{~s}\right)$, whereas the fluorescence may follow the excitation by many nanoseconds, what causes the experimental and interpretative difficulties, especially when relaxation and intermolecular energy transfer processes occur.

The intensity of the fluorescence excited with the light beam polarized in the $i$-direction and observed after passing the analyzer giving the light polarized along $j$-axis is given by

$$
J_{i j}=\left\langle M_{\mathrm{a} i}^{2} \cdot M_{\mathrm{ej}}^{2}\right\rangle,
$$

where $\boldsymbol{M}_{\mathrm{a} i}$ and $\boldsymbol{M}_{\mathrm{e} j}$ are the absorption and emission oscillator components projected onto the axes $i$ and $j$, respectively. The average is calculated over all molecules in the illuminated volume and includes all possible angular positions of the absorption and emission oscillators weighted by the appropriate statistical distribution.

From the fluorescence intensity measurements the emission anisotropies, $R_{1}$ and $R_{2}$, for the excitation with the light polarized, respectively, parallel (Fig. 1a) and perpendicular (Fig. $1 \mathrm{~b}$ ) to the orientation axis $z$ can be calculated using following formulae:

$$
R_{1}=\frac{J_{z z}-J_{z y}}{J_{z z}+2 J_{z y}}
$$

and

$$
R_{2}=\frac{J_{y z}-J_{y y}}{J_{y z}+2 J_{y y}}
$$

$J_{i j}$ are here the reduced intensities, as they regard the correction due to instrumental, concentration and volume factors.

If the experiment is realized in such conditions that the rotational relaxation time $\tau_{\mathrm{R}}$ is much longer than the lifetime $\tau_{\mathrm{F}}$ of the excited state of the fluorescent molecule and the effect of the thermal motion on the fluorescence depolarization can be neglected, then $R_{1}$ and $R_{2}$ are related with the order parameters, $\left\langle P_{2}\right\rangle$ and $\left\langle P_{4}\right\rangle$, in the following way $[33,34]$ :

$$
\begin{aligned}
& R_{1}=\frac{\frac{1}{3}\left\langle P_{2}\right\rangle P_{2}(\cos (\beta-\delta))+A+B\left\langle P_{2}\right\rangle+6 C\left\langle P_{4}\right\rangle}{\frac{1}{3}+\frac{2}{3}\left(P_{2}\right\rangle P_{2}(\cos \beta)}, \\
& R_{2}=\frac{\frac{1}{3}\left\langle P_{2}\right\rangle P_{2}(\cos (\beta-\delta))-\frac{1}{2}\left(2 A+7 C\left\langle P_{4}\right\rangle\right)}{\frac{1}{3}-\frac{1}{3}\left\langle P_{2}\right\rangle P_{2}(\cos \beta)+A-B\left\langle P_{2}\right)+C\left\langle P_{4}\right\rangle},
\end{aligned}
$$

where $\delta$ is the angle between the absorption and emission oscillators and $\beta$ is the angle between the vector of the absorption transition moment and the long molecular axis of the dye. $A, B$ and $C$ are functions depending only on the angle $\delta$ and $\beta$. 
The second-rank order parameter $\left\langle P_{2}\right\rangle$ can also be evaluated from the polarized absorption spectra of the dye dissolved in the anisotropic matrix using the following formula [35-37]:

$$
\left\langle P_{2}\right\rangle=\frac{A_{\|}-A_{\perp}}{A_{\|}+2 A_{\perp}} \frac{1}{1-\frac{3}{2} \sin ^{2} \beta},
$$

where $A_{\|}$and $A_{\perp}$ denote the components of the absorbance polarized in parallel and perpendicularly to the director $n$.

Knowing $\delta$ from the independent experiment, the three unknown values: $\beta$, $\left\langle P_{2}\right\rangle$ and $\left\langle P_{4}\right\rangle$ can be obtained solving Eqs. (6)-(8).

\section{Results}

Table presents the temperatures of the solid-smectic A ( $\left.T_{\mathrm{CS}}\right)$, smectic-nematic $\left(T_{\mathrm{SN}}\right)$ and nematic-isotropic $\left(T_{\mathrm{NI}}\right)$ phase transitions for pure $8 \mathrm{CB}$ and $8 \mathrm{CB}$ doped with the dyes.

\section{TABLE I}

Phase transition temperatures

\begin{tabular}{c|c|c|c}
\hline \hline Sample & $T_{\mathrm{CS}}[\mathrm{K}]$ & $T_{\mathrm{SN}}[\mathrm{K}]$ & $T_{\mathrm{NI}}[\mathrm{K}]$ \\
\hline 8CB & 294.5 & 306.5 & 313.5 \\
8CB + dye I & 294.3 & 309.2 & 316.4 \\
8CB + dye II & 294.2 & 309.3 & 317.5
\end{tabular}

The angle $\beta$ was calculated from Eqs. (6)-(8) on the basis of the experimental data of the fluorescence intensities and the absorbance of the appropriate polarized components measured at the temperature just after $T_{\mathrm{CS}}$ using method described in [34]. We have supposed that in this state, because of its high viscosity, the thermal molecular motions are strongly hindered and the rotational relaxation time is much greater than the lifetime of the excited state of the fluorescent guest molecule. Therefore in this case, $\left\langle P_{2}\right\rangle$ obtained from the fluorescence measurement must be equal to that determined on the basis of the polarized absorption spectra. We have obtained the values of $\beta$ being equal to $22^{\circ}$ for the dye I and $19.5^{\circ}$ for the dye II. Knowing the value of the angle $\beta,\left\langle P_{2}\right\rangle$ and $\left\langle P_{4}\right\rangle$ order parameters from Eqs. (6) and (7) on the basis of the polarized fluorescence measurements $\left(\left\langle P_{2}\right\rangle_{\mathrm{F}},\left\langle P_{4}\right\rangle_{\mathrm{F}}\right)$ were calculated. Additionally, $\left\langle P_{2}\right\rangle_{\mathrm{A}}$ was estimated independently from Eq. (8) using the polarized absorption data $\left(\left\langle P_{2}\right\rangle_{\mathrm{A}}\right)$. For calculations, the values of the absorbance and fluorescence intensity were taken at the wavenlengths corresponding to the maxima of the absorption and emission bands, respectively. The angle $\delta$ between the absorption and emission oscillators of the dyes investigated, which is needed for the evaluation of the order parameters from the fluorescence spectra was taken from the paper [33].

Figures 3 and 4 show the order parameters $\left\langle P_{2}\right\rangle_{F}$ (open circles), $\left\langle P_{4}\right\rangle_{F}$ (solid circles) and $\left\langle P_{2}\right\rangle_{\mathrm{A}}$ (crosses) as a function of the reduced temperature $T^{*}\left(T^{*}=\right.$ $\left.T / T_{\mathrm{NI}}\right)$ for $8 \mathrm{CB}$ doped with the dye I and the dye II, respectively. 


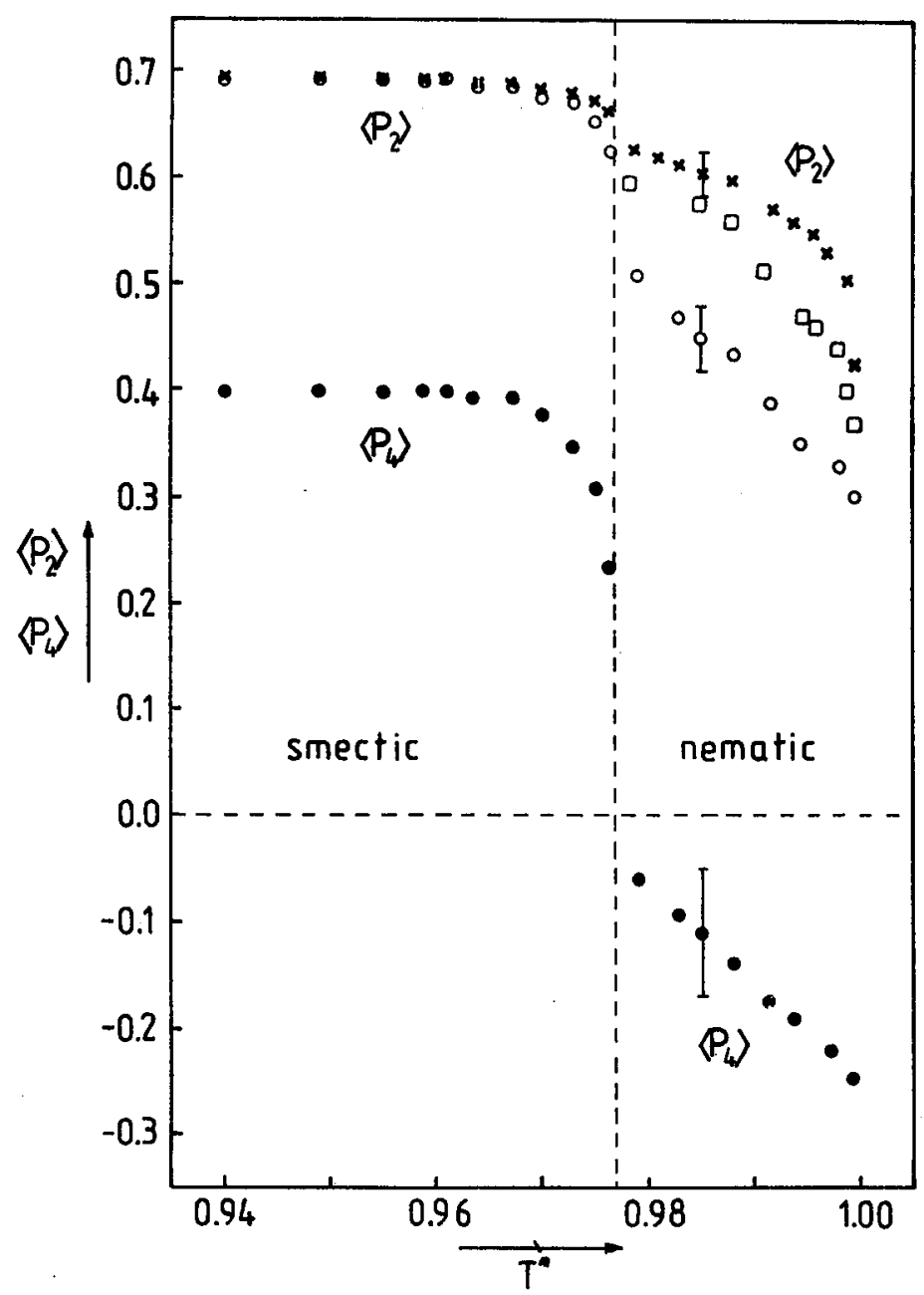

Fig. 3. $\left\langle P_{2}\right\rangle_{\mathrm{A}}$ (crosses) measured at $\lambda_{\max }^{\mathrm{A}}=437 \mathrm{~nm}$ and $\left\langle P_{2}\right\rangle_{\mathrm{F}}$ (open circles) and $\left\langle P_{4}\right\rangle_{\mathrm{F}}$ (solid circles) measured at $\lambda_{\max }^{\mathrm{F}}=550 \mathrm{~nm}$ vs. reduced temperature for $8 \mathrm{CB}$ doped with dye I. Squares represent the values of $\left\langle P_{2}\right\rangle$ for pure $8 \mathrm{CB}[38,39]$.

The squares in Figs. 3 and 4 represent the values of $\left\langle P_{2}\right\rangle$ for pure $8 \mathrm{CB}$ in the nematic phase estimated on the basis of the refractive indices measurements $[38,39]$ by the use of the Vuks approximation [40].

Knowing $\left\langle P_{2}\right\rangle$ and $\left\langle P_{4}\right\rangle$ parameters from the Eq. (1) one can obtain the truncated distribution function $f_{4}(\vartheta)$, where

$$
f_{4}(\vartheta)=\frac{1}{2}+\frac{5}{2}\left\langle P_{2}\right\rangle P_{2}+\frac{9}{2}\left\langle P_{4}\right\rangle P_{4} .
$$

In Fig. 5 the distribution functions $f_{4}(\vartheta)$ for 8 CB doped with the dye II in the smectic $\left(T^{*}=0.951\right)$ and nematic $\left(T^{*}=0.976\right)$ phases are plotted. 


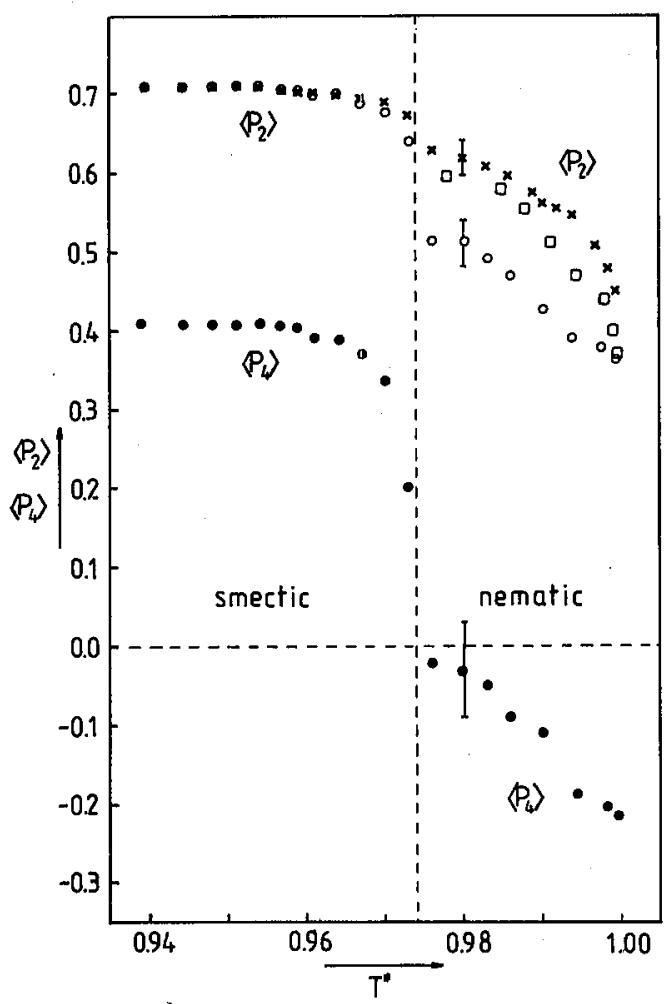

Fig. 4. $\left\langle P_{2}\right\rangle_{\mathrm{A}}$ (crosses) measured at $\lambda_{\max }^{\mathrm{A}}=436 \mathrm{~nm}$ and $\left\langle P_{2}\right\rangle_{\mathrm{F}}$ (open circles) and $\left\langle P_{4}\right\rangle_{\mathrm{F}}$ (solid circles) measured at $\lambda_{\max }^{F}=550 \mathrm{~nm}$ vs. reduced temperature for $8 \mathrm{CB}$ doped with dye II. Squares represent the values of $\left\langle P_{2}\right\rangle$ for pure 8CB [34, 35].

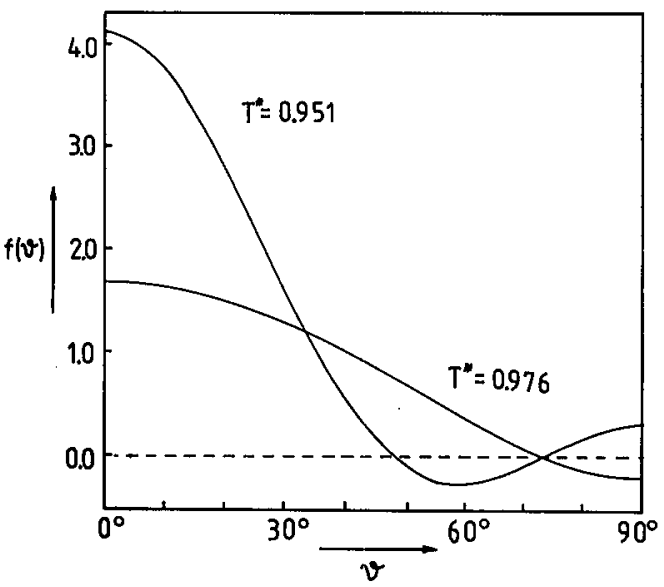

Fig. 5. Molecular distribution function $f_{4}(\vartheta)$ for $8 \mathrm{CB}$-dye II mixture in smectic $\left(T^{*}=\right.$ $0.951)$ and nematic $\left(T^{*}=0.976\right)$ phases. 


\section{Discussion}

\subsection{Phase transition temperatures}

From Table it is seen that the addition of the guest to the liquid crystal host lowers somewhat the temperature of the melting point, what is in agreement with the thermodynamic laws. However, both the smectic A-nematic and nematic-isotropic transitions occur at higher temperature in the presence of the dye as compared to pure $8 \mathrm{CB}$. The influence of the dye on the nematic-isotropic transition had been extensively investigated previously [38, 41-45]. It had been found that the direction and the magnitude of the shift in the clearing temperature of the liquid crystal after addition of the dye are strictly related to the molecular geometry of the guest and its compatibility with the anisotropic host $[38,44,45]$. In our case the elongated molecules of the dyes cause the increase in the $T_{\mathrm{NI}}$ temperature, what is in agreement with the previous results [44]. From the data gathered in Table it is seen that the dyes used in our experiment increase also the smectic A-nematic transition temperature.

\subsection{Molecular orientation}

It follows from Figs. 3 and 4 that the long orientational order in the guest-host mixture changes with the temperature, but in the smectic A phase it is not so strong function of $T$ as in the nematic one. Moreover, it is seen that the $\left\langle P_{2}\right\rangle$ order parameters estimated from the absorption $\left(\left\langle P_{2}\right\rangle_{\mathrm{A}}\right)$ and fluorescence $\left(\left\langle P_{2}\right\rangle_{\mathrm{F}}\right)$ results are equal to each other only to the certain temperature in the smectic phase. In the vicinity of the smectic A-nematic transition the both values begin to differ and this difference increases strongly in the nematic phase. This phase is in a less degree viscous than the smectic $A$ one and thus the thermal molecular motions cannot be neglected. It is impossible to assume anymore that $\tau_{\mathrm{R}} \gg \tau_{\mathrm{F}}$. The values of the both times become now to be comparable. This can lead to a considerable misinterpretation of the fluorescence emission data and cause the difference between $\left\langle P_{2}\right\rangle_{\mathrm{A}}$ and $\left\langle P_{2}\right\rangle_{\mathrm{F}} .\left\langle P_{2}\right\rangle_{\mathrm{A}}$ seems to be the more real reflection of the long orientational order in the nematic phase of the guest-host mixtures.

The results presented in Figs. 3 and 4 indicate that $\left\langle P_{2}\right\rangle_{\mathrm{A}}$ for $8 \mathrm{CB}$ doped with the dyes investigated at given temperature is greater than that for pure $8 \mathrm{CB}$ estimated from the refractive index data. It happens often when the length of the guest exceeds that of the host $[46,47]$ and it can be explained in term of the tendency of the guest molecules to balance out the more extreme deviations of the smaller host molecules from $n$. The lengths of the dye I and II molecules calculated from the known bond lengths and configuration in molecules [48] including the van der Waals radii of the terminal atoms [49] are equal to about $28 \AA$ and $31.5 \AA$, respectively. However, the lengths of the monomer $8 \mathrm{CB}$ in the most extended form is equal to $23 \AA$ [50] and if one considers the flexibility of the alkyl chain, this value reduces to about $18 \AA$ [38]. Furthermore, it is seen from Figs. 3 and 4 that the difference between $\left\langle P_{2}\right\rangle_{\mathrm{A}}$ for the guest-host mixture and $\left\langle P_{2}\right\rangle$ for the pure host increases as the temperature rises. This means that the dyes used in our 
experiments are more resistant to the thermal fluctuations compared to the host liquid crystal. This confirms the results obtained previously for azo-dyes [44].

The difference between the values of the order parameters $\left(\left\langle P_{2}\right\rangle_{\mathrm{A}}\right.$ and $\left\langle P_{2}\right\rangle_{\mathrm{F}}$ as well as $\left.\left(P_{4}\right\rangle_{\mathrm{F}}\right)$ for $8 \mathrm{CB}$-dye I mixture and those for $8 \mathrm{CB}$-dye II mixture at the given temperature is not significant and is caused by the various length of the both dyes. The values for the mixture with longer guest molecule (dye II) are always somewhat higher.

As regards $\left\langle P_{4}\right\rangle$, we can see from Figs. 3 and 4 that this order parameter is positive in the smectic phase and becomes negative in the nematic one. The negative values of $\left\langle P_{4}\right\rangle$ in the nematic phase are not in agreement with any theoretical predictions: neither with the "classical" Maier-Saupe [51] nor with the Humphries-James-Luckhurst [48] theories of nematics. Nevertheless the non-typical behaviour of the $\left\langle P_{4}\right\rangle$ parameter for some nematics had been noticed by many authors studying the orientational order by Raman scattering [27, 29-32] as well as by depolarization of fluorescence $[7,14]$. If all systematic experimental errors are estimated (as it was done in this work using the appropriate geometry and incorporating the optical corrections), then three reasons of the deviation of the experimentally obtained $\left\langle P_{4}\right\rangle$ values from the theories are possible: 1) molecular flexibility, especially due to the alkyl chain of the liquid crystal $[27,29,30], 2)$ the anisotropy of the molecular local field [28-30] and 3) the molecular association ("dimerization") due, for example, to the strongly polar-CN group of the nematic molecules [29-31]. Dalmolen et al. [26] studying the order parameters of homologous series of alkylcyanobiphenyls gave some evidences that the anomalously low values observed for $\left\langle P_{4}\right\rangle$ of some nematics might be related to the dimerization. Then one can expect two effects: 1 ) the long molecular axis of the dimer is not parallel to the direction of the Raman vibration anymore and 2) steric interactions lead to a non-parallelism of the long axis of the dimer and of a neighbouring monomer. However, it was shown in [14] that the non-typical behaviour of $\left\langle P_{4}\right\rangle$ cannot be always explained by the molecular association. The question has not been answered up to now and needs further investigations.

The negative values of $\left\langle P_{4}\right\rangle$ obtained in our paper for the nematic phase of the dye-8CB mixtures influence the wideness of the molecular distribution function, what is illustrated in Fig. 5. We can see that $f_{4}(\vartheta)$ at the temperature corresponding to the nematic phase is significantly broadened with respect to $f_{4}(\vartheta)$ determined in the smectic phase.

\section{References}

[1] G.R. Luckhurst, in: The Molecular Physics of Liquid Crystals, Eds. G.R. Luckhurst, G.W. Gray, Academic Press, London, New York, San Francisco 1979, Ch. 4 and references therein.

[2] G. Vertogen, W.H. de Jeu, Thermotropic Liquid Crystals, Fundamentals, Springer-Verlag, Berlin, Heidelberg 1988.

[3] W.L. McMillan, Phys. Rev. A 4, 1238 (1971).

[4] A.J. Leadbetter, in: The Molecular Physics of Liquid Crystals, Eds. G.R. Luckhurst, G.W. Gray, Academic Press, London, New York, San Francisco 1979, Ch. 15. 
[5] W. Haase, Z.X. Fan, H.J. Müller, J. Chem. Phys. 89, 3317 (1988).

[6] L.M. Blinov, V.A. Kiziel, V.G. Rumyancev, V.V. Titov, Kristallografiya 20, 1245 (1975).

[7] L.L. Chapoy, D.B. Du Pre, E.T. Samulski, in: Liquid Crystals and Ordered Fluids, Eds. J.F. Johnson, R.S. Porter, Plenum Press, New York, London 1978.

[8] L.L. Chapoy, D.B. Du Pre, J. Chem. Phys. 69, 519 (1978).

[9] V.K. Dolganov, B.N. Bolotin, Mol. Cryst. Liq. Cryst. 47, 197 (1978).

[10] I.N. Dozov, I.I. Penchev, J. Lumin. 22, 69 (1980).

[11] H. Yoshida, S. Kobinata, S. Maeda, Mol. Cryst. Liq. Cryst. 131, 209 (1985).

[12] A.D.L. Chandani, S. Kobinata, Mol. Cryst. Liq. Cryst. 172, 57 (1989).

[13] D. Bauman, E. Wolarz, Mol. Cryst. Liq. Cryst. Lett. 7, 161 (1990).

[14] E. Wolarz, D. Bauman, Mol. Cryst. Liq. Cryst. 197, 1 (1991).

[15] E. Wolarz, D. Bauman, J. Polym. Sci., submitted to publication.

[16] G.R. Desper, J. Kimura, J. Chem. Phys. 38, 4225 (1967).

[17] S. Nomura, H. Kawai, I. Kimura, M. Kagiyama, J. Polym. Sci. A- 28, 383 (1970).

[18] V. Zvetkoff, Acta Physicochem. (USSR) 16, 1321 (1942).

[19] A.J. Leadbetter, E.K. Norris, Mol. Phys. 38, 669 (1979).

[20] A.J. Leadbetter, P.G. Wrighton, J. Phys. (Paris) 40, C3-234 (1979).

[21] B. Bhattacharjee, S. Paul, R. Paul, Mol. Phys. 44, 1391 (1981).

[22] B. Bhattacharjee, S. Paul, R. Paul, Mol. Cryst. Liq. Cryst. 89, 181 (1982).

[23] D. Bauman, Z.X. Fan, W. Haase, Liq. Cryst. 6, 239 (1989).

[24] W. Emsley, G.R. Luckhurst, Mol. Phys. 35, 1499 (1978).

[25] S. Jen, N.A. Clark, P.S. Pershan, E.B. Priestley, J. Chem. Phys. 66, 4635 (1977).

[26] K. Miyano, J. Chem. Phys. 69, 4807 (1978).

[27] S. Prasad, S. Venugopalan, J. Chem. Phys. 75, 3033 (1981).

[28] R. Seeliger, H. Haspeklo, F. Noack, Mol. Phys. 49, 1039 (1983).

[29] L.G.P. Dalmolen, W.H. de Jeu, J. Chem. Phys. 78, 7353 (1983).

[30] L.G.P. Dalmolen, E. Egberts, W.H. de Jeu, J. Phys. (Paris) 45, 129 (1984).

[31] L.G.P. Dalmolen, S.J. Picken, A.F. de Jong, W.H. de Jeu, J. Phys. (Paris) 46, 1443 (1985).

[32] M. Kozielski, D. Bauman, M. Drozdowski, Z. Salamon, Mol. Cryst. Liq. Cryst. 142, 1 (1987).

[33] C. Zanoni, Mol. Phys. 38, 1813 (1979).

[34] E. Wolarz, Z. Nat.forsch., submitted for publication.

[35] E.D. Cehelnik, R.B. Cundall, C.J. Timmons, R.M. Bowley, Proc. R. Soc. Lond. A 335, 387 (1973).

[36] R.W. Filas, M.M. Labes, J. Appl. Phys. 52, 3949 (1981).

[37] A.V. Ivashchenko, O.S. Petrova, V.V. Titov, Mol. Cryst. Liq. Cryst. 108, 51 (1984).

[38] D. Bauman, Mol. Cryst. Liq. Cryst. 172, 41 (1989).

[39] D. Bauman, unpublished data.

[40] M.F. Vuks, Opt. Spectrosk. 20, 644 (1966). 
[41] F.C. Saunders, L. Wright, M.G. Clark, in: Liquid Crystals and Ordered Fluids, Eds. A.G. Griffin, J.F. Johnson, Plenum Press, New York, London 1984.

[42] P. Diot, J.K. Foitzik, W. Haase, Rev. Phys. Appl. 20, 121 (1985).

[43] W. Haase, O. Trinquet, U. Quotschalla, J.K. Foitzik, Mol. Cryst. Liq. Cryst. 148, 15 (1987).

[44] D. Bauman, Mol. Cryst. Liq. Cryst. 159, 197 (1988).

[45] D. Bauman, G. Czechowski, J. Jadżyn, Acta Phys. Pol. A75, 697 (1989).

[46] R. Eidenschink, Kontakte (Darmstadt) 2, 25 (1984).

[47] J. Cognard, T. Hieu Phan, Mol. Cryst. Liq. Cryst. 68, 207 (1981).

[48] Tables of Interatomic Distances and Configuration in Molecules and Ions, The Chemical Society, Burlington House, London 1958.

[49] A. Bondi, J. Phys. Chem. 68, 441 (1964).

[50] J.E. Lydon, C.J. Coakley, J. Phys. (Paris) 36, C1-45 (1975).

[51] W. Maier, A. Saupe, Z. Nat.forsch. A 13, 5564 (1958); 14, 882 (1959); 15, 287 (1960); 16, 262 (1961).

[52] R.L. Humphries, P.G. James, G.R. Luckhurst, J. Chem. Soc. Faraday Trans. II 68, 1031 (1972). 